\title{
On cusped solitary waves in finite water depth
}

\author{
Shijun Liao \\ State Key Laboratory of Ocean Engineering \\ School of Naval Architecture,Ocean and Civil Engineering \\ Shanghai Jiaotong University, Shanghai 200240, China \\ Department of Mathematics, Shanghai Jiaotong University, Shanghai 200240, China \\ ( Email address: sjliao@sjtu.edu.cn )
}

\begin{abstract}
It is well-known that the Camassa-Holm $(\mathrm{CH})$ equation admits both of the peaked and cusped solitary waves in shallow water. However, it was an open question whether or not the exact wave equations can admit them in finite water depth. Besides, it was traditionally believed that cusped solitary waves, whose 1st-derivative tends to infinity at crest, are essentially different from peaked solitary ones with finite 1st-derivative. Currently, based on the symmetry and the exact water wave equations, Liao [1] proposed a unified wave model (UWM) for progressive gravity waves in finite water depth. The UWM admits not only all traditional smooth progressive waves but also the peaked solitary waves in finite water depth: in other words, the peaked solitary progressive waves are consistent with the traditional smooth ones. In this paper, in the frame of the linearized UWM, we further give, for the first time, the cusped solitary waves in finite water depth, and besides reveal a close relationship between the cusped and peaked solitary waves: a cusped solitary wave is consist of an infinite number of peaked solitary ones with the same phase speed, so that it can be regarded as a special peaked solitary wave. This also well explains why and how a cuspon has an infinite 1st-derivative at crest. It is found that, like peaked solitary waves, the vertical velocity of a cusped solitary wave in finite water depth is also discontinuous at crest $(x=0)$, and especially its phase speed has nothing to do with wave height, too. In addition, it is unnecessary to consider whether the peaked/cusped solitary waves given by the UWM are weak solution or not, since the governing equation is not necessary to be satisfied at crest. All of these would deepen and enrich our understandings about the cusped solitary waves.
\end{abstract}

PACS: 45.50.Jf, 05.45.-a, 95.10.Ce

Key words Solitary waves, cusped crest, discontinuity

\section{Introduction}

The smooth solitary surface wave was first reported by John Scott Russell [2] in 1844. Since then, various types of solitary waves have been found. The mainstream 
models of shallow water waves, such as the Boussinesq equation [3], the KdV equation [4], the BBM equation [5] and so on, admit dispersive smooth periodic/solitary progressive waves with permanent form: the wave elevation is infinitely differentiable everywhere. Especially, the phase speed of the smooth waves is highly dependent upon wave height: the larger the wave height of a smooth progressive wave, the faster it propagates. Nowadays, the smooth amplitude-dispersive periodic/solitary waves are the mainstream of researches in water waves.

In 1993, Camassa and Holm [6] proposed the celebrated Camassa-Holm (CH) equation for shallow water waves, and first reported the so-called peaked solitary wave, called peakon, which has a peaked crest with a discontinuous (but finite) 1storder derivative at crest. This is a breakthrough in water wave theories, since it opens a new field of research in the past 20 years. Physically, different from the KdV equation and Boussinesq equation, the $\mathrm{CH}$ equation can model phenomena of not only soliton interaction but also wave breaking [7. Mathematically, the $\mathrm{CH}$ equation is integrable and bi-Hamiltonian, therefore possesses an infinite number of conservation laws in involution [6]. Besides, it is associated with the geodesic flow on the infinite dimensional Hilbert manifold of diffeomorphisms of line [7]. Thus, the $\mathrm{CH}$ equation has lots of intriguing physical and mathematical properties. It is even believed that the $\mathrm{CH}$ equation "has the potential to become the new master equation for shallow water wave theory" [8]. In addition, Kraenkel and Zenchuk [9] reported the cusped solitary waves of the $\mathrm{CH}$ equation, called cuspon. The so-called cuspon is a kind of solitary wave with the 1st derivative going to infinity at crest. Note that, unlike a peakon that has a finite 1st derivative, a cuspon has an infinite 1st derivative at crest. Thus, it was traditionally believed that peakons and cuspons are completely different two kinds of solitary waves.

However, the $\mathrm{CH}$ equation is a simplified model of water waves in shallow water. It was an open question whether or not the exact wave equations admit the peaked and cusped solitary waves in finite water depth. For example, the velocity distribution of peaked/cusped solitary waves in the vertical direction was unknown, since it can not be determined by a wave model in shallow water (such as the $\mathrm{CH}$ equation). Currently, based on the symmetry and the exact wave equations, Liao proposed a unified wave model (UWM) for progressive gravity waves in finite water depth with permanent form [1]. It was found that the UWM admits not only all traditional smooth periodic/solitary waves but also the peaked solitary waves in finite water depth, even including the famous peaked solitary waves of the $\mathrm{CH}$ equation as its special case. Therefore, the UWM unifies both of the smooth and peaked solitary waves in finite water depth, for the first time. In other words, the progressive peaked solitary waves in finite water depth are consistent with the traditional smooth waves, and thus are as acceptable and reasonable as the smooth ones.

In this article, using the linearized UWM, we give an closed-form expression of cusped solitary waves in finite water depth, and illustrate that a cusped solitary wave is consist of an infinite number of peaked solitary ones. This reveals, for the first time to the best of my knowledge, a simple but elegant relationship between the peaked and cusped solitary waves in finite water depth. 


\section{Cusped solitary waves in finite water depth}

Let us first describe the UWM briefly. Consider a progressive gravity wave propagating on a horizontal bottom in a finite water depth $D$, with a constant phase speed $c$ and a permanent form. For simplicity, the problem is solved in the frame moving with the phase speed $c$. Let $x, z$ denote the horizontal and vertical dimensionless co-ordinates (using the water depth $D$ as the characteristic length), with $x=0$ corresponding to the wave crest, $z=-1$ to the bottom, and the $z$ axis upward, respectively. Assume that the wave elevation $\eta(x)$ has a symmetry about the crest, the fluid in the interval $x>0$ is inviscid and incompressible, the flow in $x>0$ is irrotational, and surface tension is neglected. Here, it should be emphasized that, different from all traditional wave models, the flow at $x=0$ is not absolutely necessary to be irrotational. Let $\phi(x, z)$ denote the velocity potential. All of them are dimensionless using $D$ and $\sqrt{g D}$ as the characteristic scales of length and velocity, where $g$ is the acceleration due to gravity. In the frame of the UWM, the velocity potential $\phi(x, z)$ and the wave elevation $\eta(x)$ are first determined by the exact wave equations (i.e. the Laplace equation

$\nabla^{2} \phi=0$, the two nonlinear boundary conditions on the unknown free surface $\eta$, the bed condition and so on) only in the interval $x \in(0,+\infty)$, and then extended to the whole interval $(-\infty,+\infty)$ by means of the symmetry

$$
\eta(-x)=\eta(x), u(-x, z)=u(x, z), v(-x, z)=-v(x, z),
$$

which enforces the additional restriction condition $v(0, z)=0$. Note that, in the frame of the UWM, the flow at $x=0$ is not necessarily irrotational, so that the UWM is more general: this is the reason why the UWM can admit both of the smooth and peaked solitary waves.

In the interval $(0,+\infty)$, the governing equation $\nabla^{2} \phi(x, z)=0$ with the bed condition $\phi_{z}(x,-1)=0$ has two kinds of general solutions [10], where the subscript denotes the differentiation with respect to $z$. One is

$$
\cosh [n k(1+z)] \sin (n k x),
$$

corresponding to the smooth periodic waves with the dispersive relation

$$
\alpha^{2}=\frac{\tanh (k)}{k} \leq 1,
$$

where $\alpha=c / \sqrt{g D}$ is the dimensionless phase speed, $k$ is wave number and $n$ is an integer, respectively. The other is

$$
\cos [n k(z+1)] \exp (-n k x),
$$

corresponding to the peaked solitary waves in finite water depth [1], with the relation

$$
\alpha^{2}=\frac{\tan (k)}{k} \geq 1,
$$

where $k$ has nothing to do with wave number. Given $\alpha \leq 1$ for the smooth periodic waves, the transcendental equation (1) has a unique solution, as mentioned in the 
textbook [10]. However, given $\alpha \geq 1$ for the peaked solitary waves, the transcendental equation (2) has an infinite number of solutions:

$$
\alpha^{2}=\frac{\tan k_{n}}{k_{n}}, \quad n \pi \leq k_{n} \leq n \pi+\frac{\pi}{2}, \quad n \geq 0,
$$

corresponding to an infinite number of peaked solitary waves [1]

$$
\eta_{n}(x)=A_{n} \exp \left(-k_{n}|x|\right)
$$

in the frame of the linear UWM, where $A_{n}$ denotes its wave height. For example, when $\alpha^{2}=2 \sqrt{3} / \pi$, the transcendental equation (2) has an infinite number of solutions $k_{0}=\pi / 6, k_{1}=4.51413, k_{2}=7.73730, k_{3}=10.91266, k_{4}=14.07281, k_{5}=17.22616$, $k_{6}=20.37587, k_{7}=23.52341, k_{8}=26.66955, k_{9}=29.81472, k_{10}=32.95921$, and the asymptotic expression

$$
k_{n} \approx(n+0.5) \pi, \quad n>10,
$$

with less than $0.08 \%$ error. In general, $k_{n} \approx(n+0.5) \pi$ is a rather accurate approximation of $k_{n}$ for large enough integer $n$.

Obviously, the peaked solitary wave (44) is not smooth at crest, i.e. its first derivative is discontinuous. Note that the well-known peaked solitary wave $\eta=c \exp (-|x|)$ of the $\mathrm{CH}$ equation is only a special case of (4) when $A_{n}=c$ and $k_{n}=1$. However, unlike $\eta=c \exp (-|x|)$ that is a weak solution of the $\mathrm{CH}$ equation, it is unnecessary to consider whether or not the peaked solitary wave (4) is a kind of weak solution, because the $\mathrm{CH}$ equation is defined in the whole domain $-\infty<x<+\infty$ but the governing equation of the UWM is defined only in $0<x<+\infty$. Physically, unlike the $\mathrm{CH}$ equation and the fully nonlinear wave equations, waves in the frame of the UWM are not necessary to be irrotational at $x=0$, therefore the governing equation holds only in the domain $0<x<+\infty$, since the solution in the interval $-\infty<x<0$ is gained by means of the symmetry. Mathematically, $x=0$ is a boundary of the governing equation, and it is well-known that solutions of differential equations can be non-smooth at boundary, like a beam with discontinuous cross sections acted by a constant bending moment. Therefore, in the frame of the UWM, it is unnecessary to consider whether or not the peaked solitary waves (4) are weak solutions at all. This is the reason why, unlike the well-known peaked solitary wave $\eta=c \exp (-|x|)$ of the $\mathrm{CH}$ equation whose phase speed is always equal to its wave height, the phase speed of the peaked solitary waves (4) given by the UWM has nothing to do with wave height! This is the most attractive novelty of the UWM.

The above peaked solitary waves in finite water depth have some unusual characteristics, as revealed by Liao [1]. First, it has a peaked crest with a discontinuous vertical velocity $v$ at crest. Besides, unlike the smooth waves whose horizontal velocity $u$ decays exponentially from free surface to bottom, the horizontal velocity $u$ of the peaked solitary waves at bottom is always larger than that on free surface. Especially, different from the smooth waves whose phase speed depends upon wave height, the phase speed of the peaked solitary waves in finite water depth has nothing to do with wave height, i.e. it is non-dispersive. 


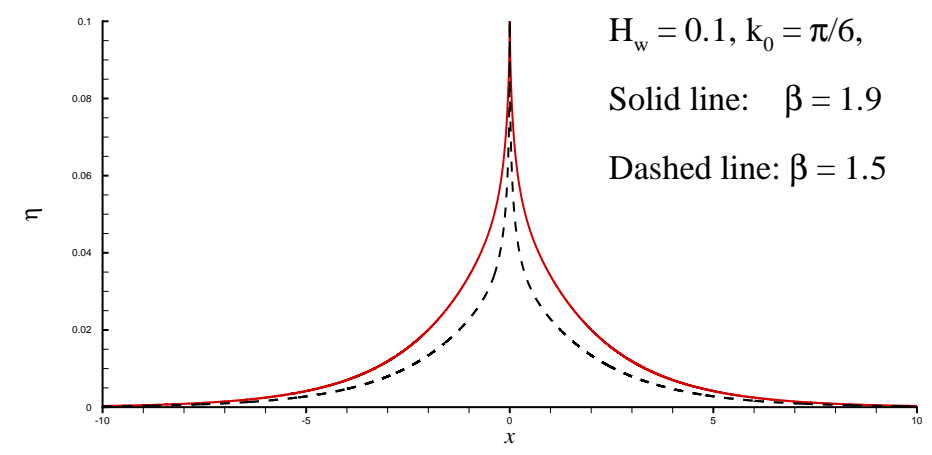

Figure 1: Cusped solitary waves in finite water depth defined by (6) when $H_{w}=0.1$ and $k_{0}=\pi / 6$ (corresponding to $\alpha=12^{1 / 4} / \sqrt{\pi}$ ). Solid line: $\beta=1.9$; Dashed line: $\beta=1.5$.

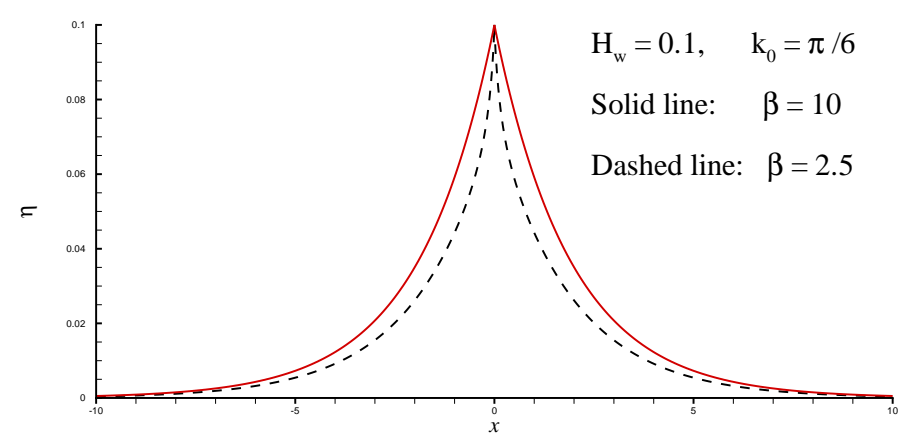

Figure 2: Peaked solitary waves in finite water depth defined by (6) when $H_{w}=0.1$ and $k_{0}=\pi / 6$ (corresponding to $\alpha=12^{1 / 4} / \sqrt{\pi}$ ). Solid line: $\beta=10$; Dashed line: $\beta=2.5$. 
Thus, in the frame of the linear UWM [1], given a dimensionless phase speed $\alpha \geq 1$, there exist an infinite number of peaked solitary waves $A_{n} \exp \left(-k_{n}|x|\right)$ with the same phase speed $\alpha$ but different wave amplitudes $A_{n}$. Thus, we may have such peaked solitary waves

$$
\eta(x)=\sum_{n=0}^{\infty} A_{n} \exp \left(-k_{n}|x|\right),
$$

where $A_{n}$ is a constant, which can be chosen with great freedom, as long as the above infinite series is convergent in the whole interval $(-\infty,+\infty)$. As a special case of it, let us consider such a one-parameter family of wave elevations

$$
\eta(x)=\frac{H_{w}}{\zeta(\beta)} \sum_{n=1}^{+\infty} \frac{1}{n^{\beta}} \exp \left(-k_{n-1}|x|\right), \quad \beta>1,
$$

where $H_{w}$ denotes wave height, $\beta>1$ is a constant, $\zeta(\beta)$ is the Riemann zeta function, and $k_{n}$ is determined by (2) for the given $\alpha \geq 1$, respectively. Since $\beta>1$, we have $\sum_{n=1}^{+\infty} n^{-\beta}=\zeta(\beta)$ so that the above infinite series converges to the wave height $H_{w}$ at $x=0$, and besides is convergent in the whole interval $(-\infty,+\infty)$. However, its 1st derivative at $x=0$, i.e.

$$
\eta^{\prime}(0)= \pm \frac{H_{w}}{\zeta(\beta)} \sum_{n=1}^{+\infty} \frac{k_{n-1}}{n^{\beta}},
$$

is convergent to a finite value when $\beta>2$, but tends to infinity when $1<\beta \leq 2$, because $k_{n-1} \approx(n-0.5) \pi$ for large enough integer $n$ and the series $\sum 1 / n^{\beta-1}$ is convergent when $\beta>2$ but tends to infinity when $0<\beta \leq 2$. So, the infinite series (6) defines a cusped solitary wave in finite water depth when $1<\beta \leq 2$ and a peaked solitary wave when $\beta>2$. Therefore, in essence, a cusped solitary wave in finite water depth is consist of an infinite number of peaked solitary waves (when $1<\beta \leq 2$ ) with the same phase speed! To the best of the author's knowledge, this reveals, for the first time, a simple but elegant relationship between the peaked and cusped solitary waves in finite water depth! In addition, the infinite series (6) illustrates the consistency of the peaked and cusped solitary waves, and besides explains why and how a cuspon has an infinite 1st-derivative at crest. Since the phase speed of peaked solitary waves (4) in finite water depth has nothing to do with the wave height, it is straight forward that the phase speed of a cusped solitary wave in finite water depth also has nothing to do with the wave height, too.

Note that, according to the definition of the wave elevation (6), given a dimensionless phase velocity $\alpha \geq 1$ and an arbitrary wave height $H_{w}$, there exist an infinite number of cusped solitary waves, dependent upon $\beta \in(1,2]$. For example, the two cusped solitary waves in finite water depth defined by the infinite series (6) in the case of $\alpha^{2}=2 \sqrt{3} / \pi, H_{w}=1 / 10$ when $\beta=1.5$ and $\beta=1.9$ are as shown in Fig. 1. It should be emphasized that the same expression (6) can define an infinite number of peaked solitary waves in finite water depth, too, depending on $\beta \in(2,+\infty)$. For example, the two peaked solitary waves in finite water depth in the case of $\alpha^{2}=2 \sqrt{3} / \pi, H_{w}=1 / 10$ when $\beta=5 / 2$ and $\beta=10$ are as shown in Fig. 2. This well illustrates the consistency of the peaked and cusped solitary waves in finite water depth. 
Theoretically speaking, given an arbitrary wave height $H_{w}$ and a dimensionless phase speed $\alpha \geq 1$, there are many different types of peaked/cusped solitary waves in finite water depth. For example, a more generalized, two-parameter family of peaked/cusped solitary waves in finite water depth reads

$$
\eta(x)=\frac{H_{w}}{\zeta(\beta, \gamma)} \sum_{n=0, n \neq-\gamma}^{+\infty} \frac{1}{(n+\gamma)^{\beta}} \exp \left(-k_{n}|x|\right),
$$

where $\beta>1$ and $\gamma \neq 0$ are constants to be chosen with great freedom, $\zeta(\beta, \gamma)$ is a generalized Riemann zeta function, and $k_{n}$ is determined by (2) for the given $\alpha \geq 1$, respectively. Since $k_{n} \approx(n+0.5) \pi$ for large enough integer $n$, the above infinite series defines a cusped solitary wave when $1<\beta \leq 2$ and a peaked ones when $\beta>2$, respectively. This illustrates once again the consistency of the peaked and cusped solitary waves in finite water depth.

According to the linearized UWM [1], the velocity potential $\phi^{+}$(defined only in the interval $x>0$ ) corresponding to the peaked/cusped solitary wave elevation (6) reads

$$
\phi^{+}=-\frac{\alpha H_{w}}{\zeta(\beta)} \sum_{n=1}^{+\infty} \frac{\cos \left[k_{n-1}(z+1)\right] \exp \left(-k_{n-1} x\right)}{n^{\beta} \sin \left(k_{n-1}\right)},
$$

which gives, using the symmetry, the corresponding horizontal velocity

$$
u=\frac{\alpha H_{w}}{\zeta(\beta)} \sum_{n=0}^{+\infty} \frac{k_{n} \cos \left[k_{n}(z+1)\right] \exp \left(-k_{n}|x|\right)}{(n+1)^{\beta} \sin \left(k_{n}\right)}
$$

in the whole interval $x \in(-\infty,+\infty)$, the vertical velocity

$$
v^{+}=\frac{\alpha H_{w}}{\zeta(\beta)} \sum_{n=0}^{+\infty} \frac{k_{n} \sin \left[k_{n}(z+1)\right] \exp \left(-k_{n} x\right)}{(n+1)^{\beta} \sin \left(k_{n}\right)}
$$

in the interval $x \in(0,+\infty)$, and the vertical velocity

$$
v^{-}=-\frac{\alpha H_{w}}{\zeta(\beta)} \sum_{n=0}^{+\infty} \frac{k_{n} \sin \left[k_{n}(z+1)\right] \exp \left(k_{n} x\right)}{(n+1)^{\beta} \sin \left(k_{n}\right)}
$$

in the interval $(-\infty, 0)$, respectively. Obviously, it holds

$$
\lim _{x \rightarrow 0} v^{+}=-\lim _{x \rightarrow 0} v^{-}
$$

for the cusped $(1<\beta \leq 2)$ and peaked $(\beta>2)$ solitary waves, although we always have $v=0$ at $x=0$. Thus, like a peakon in finite water depth, a cuspon in finite water depth has the velocity discontinuity at $x=0$, too.

Especially, at $z=0$ and as $x \rightarrow 0$, the corresponding vertical velocity reads

$$
\lim _{x \rightarrow 0} v^{+}(x, 0)=\frac{\alpha H_{w}}{\zeta(\beta)} \sum_{n=0}^{+\infty} \frac{k_{n}}{(n+1)^{\beta}},
$$


which is finite when $\beta>2$ but tends to infinity when $1<\beta \leq 2$, since $k_{n} \approx(n+0.5) \pi$ for large enough integer $n$. Thus, unlike a peaked solitary wave in finite water depth whose $v$ is always finite, the vertical velocity of the cusped solitary waves in finite water depth tends to infinity at $z=0$ as $x \rightarrow 0$. Mathematically, this is acceptable, since it is traditionally believed that a cuspon has a higher singularity than a peakon. Such kind of singularity leads to a more strong vortex sheet at $x=0$ near $z=0$. Physically, in reality such kind of singularity and discontinuity, "if it could ever be originated, would be immediately abolished by viscosity", as mentioned by Lamb [11].

\section{Concluding remarks}

In summary, in the frame of the linearized UWM [1], we give, for the first time, the cusped solitary waves in finite water depth, and reveal that a cuspon is consist of an infinite number of peaked solitary waves with the same phase speed. This kind of consistency also well explains why and how the 1st-derivative of a cusped solitary wave tends to infinity at crest. It is found that, like a peakon, the vertical velocity of a cuspon is also discontinuous at $x=0$, and besides, its phase speed also has nothing to do with wave height, too. All of these would deepen and enrich our understandings about the peaked and cusped solitary waves.

It should be emphasized that, in the frame of the UWM, the governing equation is defined only in the domain $0<x<+\infty$, since the solution at $-\infty<x<0$ is given by means of the symmetry. This is quite different from other wave equations such as the $\mathrm{CH}$ equation and the fully nonlinear wave equations, which are defined in the whole domain $-\infty<x<+\infty$. Physically, it means that the flow at crest is not absolutely necessary to be irrotational. Thus, mathematically, we need not consider whether the peaked/cusped solitary waves are weak solutions or not. This

is the reason why, unlike the well-known peaked solitary wave $\eta=c \exp (-|x|)$ of the $\mathrm{CH}$ equation, whose phase speed is always equal to wave height, the phase speed of the peaked/cusped solitary waves (4) given by the UWM has nothing to do with wave height! This is the most attractive novelty of the UWM, which provides us a simple but elegant relationship between peaked and cusped solitary waves in finite water depth.

This work is partly supported by the State Key Laboratory of Ocean Engineering (Approval No. GKZD010061) and the National Natural Science Foundation of China (Approval No. 11272209).

\section{References}

[1] Liao, S.J. Do peaked solitary water waves indeed exist? Commun. Nonlinear Sci. Numer. Simulat. published online (dpi:/10.1016/j.cnsns.2013.09.042) 
[2] Russell, J.S. Report on waves. Fourteenth Meeting of the British Association for the Advancement of Science (1844).

[3] Boussinesq, J. Théorie des ondes et des remous qui se propagent le long d'un canal rectangulaire horizontal, en communiquant au liquide contenu dans ce canal des vitesses sensiblement pareilles de la surface au fond. Journal de Math'ematiques Pures et Appliquées. Deuxiéme Série 17: 55 - 108 (1872).

[4] Korteweg, D. J. and de Vries, G. On the Change of Form of Long Waves Advancing in a Rectangular Canal, and on a New Type of Long Stationary Waves'. Phil. Mag. 39, 422 - 443 (1895)

[5] Benjamin, B., Bona, J.L. and Mahony, J.J. Model equations for long waves in nonlinear dispersive systems. Philos. Trans. Roy. Soc. London, 272: 47-78 (1972)

[6] Camassa, R. and Holm, D. D. An integrable shallow water equation with peaked solitons. Phys. Rev. Lett. 71, 1661 - 1664 (1993).

[7] Constantin, A. Existence of permanent and breaking waves for a shallow water equation: a geometric approach. Ann. Inst. Fourier, Grenoble. 50, No.2, 321 $362(2000)$

[8] Fuchssteiner, B. Some tricks from the symmetry-toolbox for nonlinear equations: generalizations of the Camassa-Holm equation. Physica D. 95, 296 - 343 (1996)

[9] Kraenkel, R. A. and Zenchuk, A. Camassa - Holm equation: transformation to deformed sinhGordon equations, cuspon and soliton solution. J. Phys. A: Math. Gen. 32, 4733 - 4747 (1999)

[10] Mei C. C., Stiassnie M. and Yue D. K. P. Theory and applications of ocean surface waves. New Jersey: World Scientific (2005)

[11] Lamb, H. Hydrodynamics. Dover Publications, New York (1932). 